\title{
Análises de dosagens de concreto asfáltico do tipo Pré Misturado a Frio (PMF) utilizando Resíduos da Construção e Demolição de obras (RCD)
}

\author{
Analysis of Cold Asphalt Concrete \\ Mixtures Using Construction and \\ Demolition Wastes
}

\footnotetext{
${ }^{1}$ Universidade Federal do Rio Grande do Norte - Programa de Pós-Graduação em Engenharia Civil - (PEC - UFRN), Av. Sen. Salgado Filho, 3000, 59064-741, Candelária, Natal, RN, Brasil.

e-mail: placidogsn@ hotmail.com, enio.amorim@ifrn.edu.br, pilarduranteingunza@gmail.com
}

\section{RESUMO}

Na construção civil, um problema ambiental recorrente é a geração e disposição de Resíduos de Construção e Demolição (RCD) de obras. A utilização do RCD como agregados na composição de concreto asfáltico, do tipo Pré Misturado a Frio (PMF), torna possível a execução de pavimentos rodoviários de baixo tráfego. Diante desses fatos, este artigo propõe avaliar a viabilidade técnica de materiais reciclados, por meio de ensaios de caracterização laboratoriais e dosagens de concreto asfáltico, substituindo agregados naturais por reciclados, em misturas de concreto asfáltico a serem utilizadas como camada de revestimento em um pavimento. Na pesquisa, foram realizadas análises de caracterização física do material reciclado; caracterização química e mineralógica do fíler RCD (DRX, FRX, EDS e MEV); e análises de dosagens Marshall do concreto asfáltico do tipo PMF, variando os teores de emulsão asfáltica (EAP) em 6\%, 7\%, 8\% e 9\%, em cada concepção proposta no estudo. Os resultados químicos e mineralógicos indicaram teores significativos de sílica, ferro e alumínio, caracterizando a origem predominantemente de cerâmica no material do RCD. Analisando-se os parâmetros volumétrcios da metodologia Marshall, verificou-se a interação de teores de emulsão com as propriedades de estabilidade, volume de vazios, densidade e relação betume vazios, constatando-se um desempenho inferior do concreto com agregados RCD, porém, condizentes com os parâmetros aceitáveis da norma Marshall de dosagem. Desse modo, foi possível constatar o expressivo potencial (econômico e ambiental) do uso do RCD na composição de concretos asfálticos a frio, como camadas de revestimento asfáltico, em pavimentos de baixo tráfego.

Palavras-chave: Agregados reciclados, Pavimentação asfáltica, Camadas de revestimento.

\section{ABSTRACT}

In civil construction, a recurring environmental problem is the generation and disposal of Construction and Demolition Waste (RCD) of works. The use of RCD as aggregates of pre-mixed mixed asphalt concrete (PMF) makes possible the implementation of low traffic pavements. Therefore, this article proposes to evaluate the technical feasibility of the recycled materials, through laboratory tests and dosages of asphalt concrete, replacing natural aggregates by recycled in mixtures of asphalt concrete to be used as floor covering layer. In the research, analyzes of physical characterization of the material were carried out; mineralogical characterization of the RCD filler (DRX, FRX, EDS and MEV); and analyzes of Marshall's dosages of asphalt concrete (Pre-mixed to PMF) varying in asphalt emulsion contents in $6 \%, 7 \%, 8 \%$ and $9 \%$, in each mixing design. The FRX results indicated oxides such as $\mathrm{SiO}_{2}, \mathrm{Fe}_{2} \mathrm{O}_{3}$ and $\mathrm{Al}_{2} \mathrm{O}_{3}$, with significant silica, iron and aluminum contents, also characterizing the predominantly ceramic origin of the RCD material. Analyzing Marshall methodology parameters, the interaction of emulsion contents with Stability, Void Volume, Density, and Empty Bitumen properties showed lower performance of concrete with RCD aggregates, however, consistent with the Marshall standard parameters 
of dosage. Therefore, it was possible to verify the expressive potential (economic and environmental) of the use of RCD in the composition of cold asphalt concrete, as layers of asphalt, in low traffic pavements.

Key-words: Recycled Aggregates, Asphalt Paving, Coating Layers.

\section{INTRODUÇÃO}

Na construção civil, a disposição final do RCD constitui um problema ambiental recorrente. Dentro dos programas de gestão sustentável o uso desta classe de resíduos em misturas de concreto asfáltico é uma prática atualmente em desenvolvimento.

BERNUCCI et al. [2] reportam que existem alguns tipos usuais de revestimentos asfálticos que podem ser aplicados como uma das camadas do pavimento. São os revestimentos com misturas usinadas a quente, usinadas a frio e em usinas móveis. Com ênfase nas misturas a frio, o pré-misturado a frio (PMF) consiste nas misturas de agregados (graúdos, miúdos e de materiais finos) com a emulsão asfáltica de petróleo. Os autores destacam que o uso de emulsões do tipo Ruptura Lenta (RL) em concretos asfálticos utilizando agregados de maiores resistências físicas propicia um desempenho eficaz no concreto asfáltico.

De acordo com LEITE et al. [8], para efeitos de análise característica do RCD, existe um decréscimo com relação a capacidade de suporte de carga do material. Esse efeito de resistência do material pode ser aferido por ensaios de caracterização geotécnica do material quando comparado com materiais nobres. Entretanto, conforme BERNUCCI et al. [2], algumas propriedades mais específicas no concreto asfáltico podem ser avaliadas de maneira comparativa, destacando-se a absorção de água no material, a rugosidade superficial, a estabilidade por capacidade de aplicação de cargas, entre outras.

FATEMI e IMANINASAB [4] avaliaram que o emprego do RCD em uma das camadas do pavimento, em determinadas proporções, melhora algumas propriedades do concreto asfáltico. Essas propriedades podem ser mensuradas por meio de análises de rastreamento de rodas na escala de campo. No entanto, de uma maneira geral, a utilização do RCD como material agregado em substituição parcial ou total do material nobre, pode acarretar em diminuição de algumas propriedades, tanto em misturas de concretos utilizando o cimento Portland, quanto em composições de concretos asfálticos.

Segundo GÓMEZ-MEIJIDE et al. [6], o concreto asfáltico usinado a frio possui algumas vantagens relacionadas à economia e ao ambiente. No entanto, esse tipo de mistura necessita de determinado tempo para rompimento do ligante. No programa experimental, os autores avaliaram as propriedades do pré-misturado a frio com agregados do tipo RCD, como também verificaram que ocorreram ganhos de rigidez e perda de água quando comparados com outras misturas.

Nos estudos realizados na China por JAMSHIDI et al. [7] sobre avaliação da capacidade térmica nas misturas de concretos asfálticos, mostrou-se que, a substituição dos agregados de alta capacidade térmica específica por materiais agregados de baixa capacidade térmica proporcionava uma diminuição linear de consumo energético durante a mistura. Portanto, há uma economia significativa de energia potencialmente capaz de abastecer milhares de famílias no país.

Neste sentido, ANTHONISSEN et al. [1], demostraram a inviabilidade tanto ambiental quanto energética da incorporação de aditivos (zeólito sintético e a cera orgânica) às misturas a fim de que possibilitasse a redução energética envolvida no procedimento à quente.

Com relação à durabilidade dos materiais, GÍMENEZ [5] conclui que alguns agregados reciclados devem ser avaliados de maneira criteriosa, pois materiais resistentes são passíveis de sofrer desintegração química quando expostos a condições ambientais desfavoráveis no pavimento.

Este trabalho tem como objetivo avaliar o desempenho físico dos agregados reciclados quando incorporados ao concreto asfáltico por meio de ensaios de caracterização física, química, mineralógica e dosagens Marshall.

\section{MATERIAIS E MÉTODOS}

\subsection{Materiais da pesquisa}

A concepção do presente trabalho empregou agregados naturais na fração de brita 1, oriundos de rocha granítica, e areia natural extraída de um leito de rio. Para efeito comparativo e avaliação do potencial técnico, utilizou-se agregados de RCD em frações similiares aos agregados naturais. 


\subsection{Metodologia empregada na pesquisa}

Para que houvesse um conhecimento mais aprofundado do material reciclado, foram realizados ensaios de caracterização química e mineralógica com a finalidade de identificar a composição química e estrutural dos grãos, bem como buscar compatibilidade com os resultados obtidos por meio de outras pesquisas correlatas. Os ensaios realizados foram: Microscopia Eletrônica de Varredura (MEV); Espectrometria de Energia Dispersiva (EDS); Difração de Raio X (DRX) e Fluorescência de Raios X (FRX).

No que diz respeito ao estudo das dosagens, para definir a melhor dosagem, foi estabelecido um traço padrão contendo todos os agregados na condição natural, e alternando os tipos dos materiais nos demais traços. Para efeito de análises, foram realizadas quatro concepções de misturas dos agregados nas dosagens. No modelo das quatro misturas dos agregados, as que tiveram brita e areia naturais teve o seu traço de dosagem denominado de D1 - BNAN; no traço que teve como composição brita natural e areia reciclada, foi chamado de D2 - BNAR; no traço que teve brita reciclada e areia reciclada, D3 - BRAR; e o traço que teve a brita reciclada e areia natural foi denominado D4 - BRAN (Tabela 1). Nas misturas que mantiveram presente a areia reciclada, por conveniência, não foi inserido o fíler natural (cimento), pois já existia quantidades consideráveis de fíler RCD no lote de agregados miúdos reciclados.

Tabela 1: Descrição dos traços utilizados.

\begin{tabular}{ccc}
\hline Descrição & Combinações de agregados & $\begin{array}{c}\text { Condição dos } \\
\text { agregados }\end{array}$ \\
\hline D1 BNAN & Brita + Areia (Naturais) & Seco \\
D2 BNAR & Brita Natural + Areia Reciclada & Seco \\
D3 BRAR & Brita Reciclada + Areia Reciclada & Seco \\
D4 BRAN & Brita Reciclada + Areia Natural & Seco \\
\hline
\end{tabular}

Em conformidade com a norma de dosagem Marshall, foram confeccionados 48 corpos de provas, para as quatro diferentes concepções de misturas dos agregados (naturais e reciclados), e quatro dosagens, compondo em cada uma delas, quatro teores de ligantes. No procedimento de confecção dos corpos de provas, após a mistura dos materiais (dosagem) e inserção em moldes, 75 golpes foram aplicados sobre o material para melhor compactá-los. Feito esse procedimento, o material foi levado à estufa por 24 horas. Com a realização de alguns ensaios laboratoriais, obteve-se os teores de ligante ótimo em cada dosagem, de acordo com os parâmetros dos resultados.

\section{RESULTADOS E DISCUSSÕES}

\subsection{Caracterização química e minaralógica do fíler - RCD}

\subsubsection{Microscopia eletrônica de varredura (MEV)}

Para uma melhor investigação de parâmetros que interferem diretamente nas propriedades do concreto asfáltico, foi realizada uma análise dos grãos cristalinos de uma pequena amostra de fíler RCD com o auxílio do ensaio de MEV, observando as seguintes características: pouca uniformidade no tamanho dos grãos (medidas em micrômetros na Figura 1); grãos com formatos e angulações arredondadas, bem como achatadas e pouco alongadas; rugosidade na superfície dos grãos e material aparentemente poroso. 


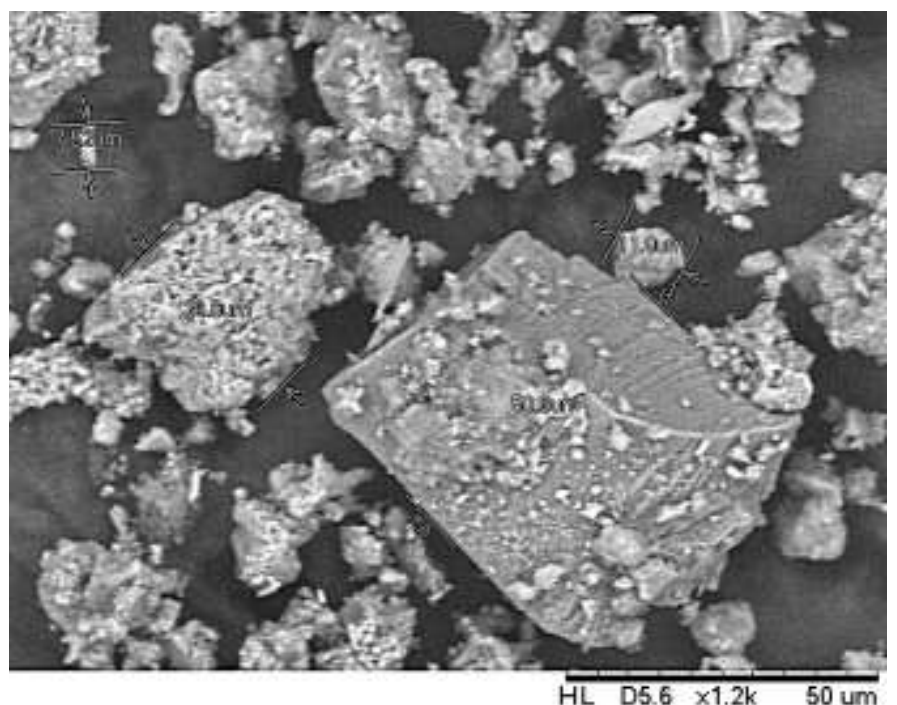

Figura 1: Varredura de grãos cristalinos com aproximação 1200x.

\subsubsection{Espectrometria por dispersão de energia de raios-x (EDS)}

Conforme procedimentos de análise do equipamento EDS acoplado junto ao MEV, alguns elementos químicos presentes na amostra de fíler RCD foram identificados em uma região previamente delimitada da amostra analisada (Figura 2).

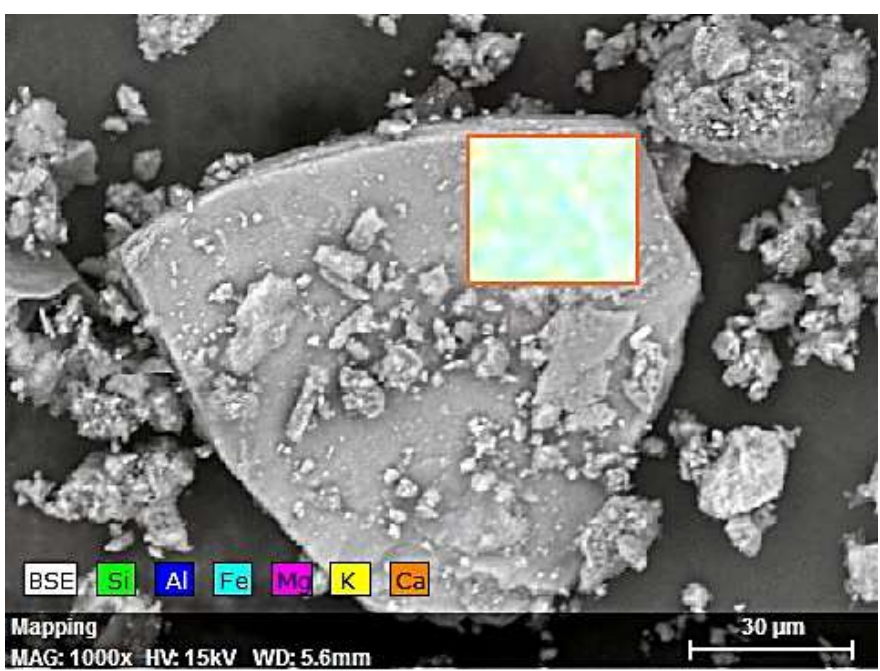

Figura 2: EDS identificando a composição química.

Na Tabela 2 encontram-se indicados quais os elementos químicos estão presentes e os seus respectivos percentuais em massa. Para tanto, foi possível constatar que os dados obtidos no EDS confirmam os compostos identificados no ensaio de FRX (Tabela 3). De acordo com esses ensaios, há percentuais significativos de silício, ferro e alumínio na amostra.

Tabela 2: Análise de EDS do fíler RCD.

\begin{tabular}{cc}
\hline ELEMENTOS & \% EM MASSA \\
\hline Alumínio & $17,86 \%$ \\
Cálcio & $2,25 \%$ \\
Magnésio & $11,63 \%$ \\
Silício & $31,41 \%$ \\
Titânio & $3,63 \%$ \\
Potássio & $9,89 \%$ \\
Ferro & $23,32 \%$ \\
\hline
\end{tabular}




\begin{tabular}{c}
\hline TOTAL \\
\hline
\end{tabular}

\subsubsection{Difração de raios-X (DRX)}

A Figura 3 mostra o difratograma do material RCD. Os minerais detectados na análise de DRX foram o Quartzo $\left(\mathrm{SiO}_{2}\right)$, Calcita $\left(\mathrm{CO}_{3} \mathrm{Ca}\right)$, Gipsita $\mathrm{Ca}\left(\mathrm{SO}_{4}\right)\left(\mathrm{H}_{2} \mathrm{O}\right)_{2}$, Caulinita $\left(\mathrm{Al}_{2} \mathrm{Si}_{2} \mathrm{O}_{5}(\mathrm{OH})_{4}\right.$ e Microclína $\mathrm{KAlSi}_{3} \mathrm{O}_{8}$. A análise da composição mineralógica do RCD se torna complexa pelo fato da grande variedade das fases cristalinas dos seus componentes.

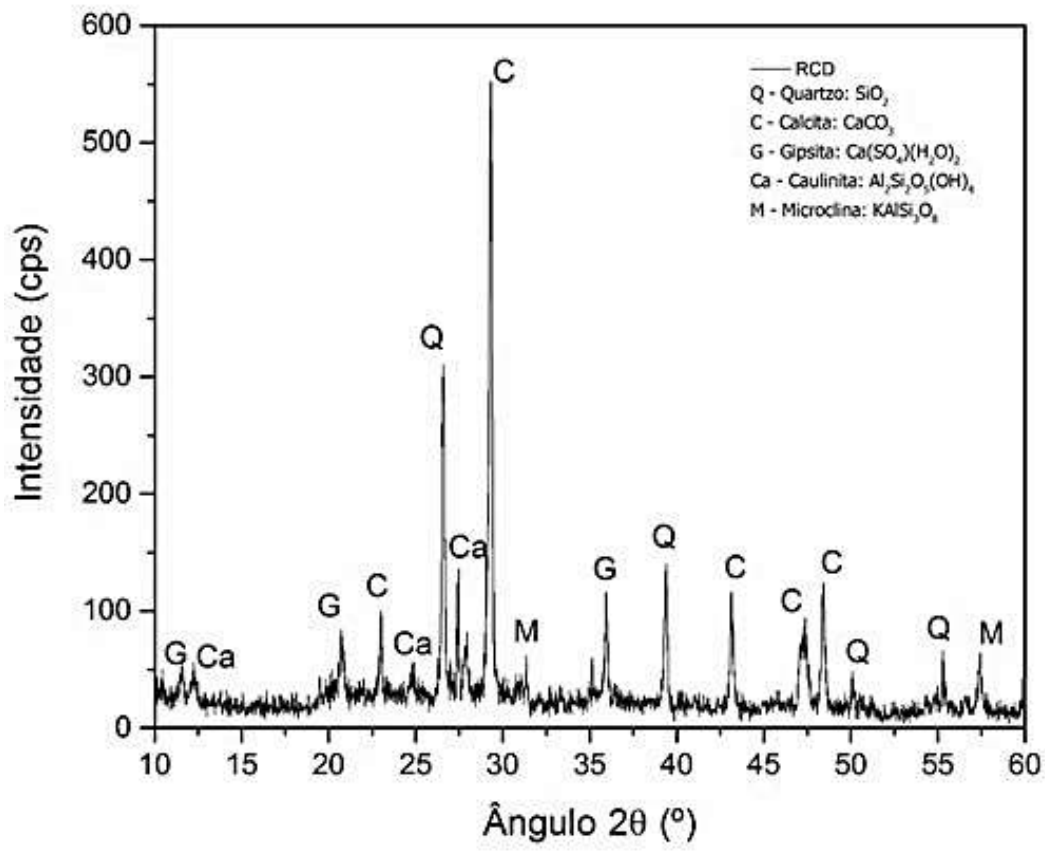

Figura 3: Difratograma da amostra de fíler RCD.

\subsubsection{Fluorescência de Raios $X$}

Com base na Tabela 3, pode-se constatar que o $\mathrm{SiO}_{2}, \mathrm{Fe}_{2} \mathrm{O}_{3}$ e $\mathrm{Al}_{2} \mathrm{O}_{3}$ são os óxidos que apresentaram percentuais significativos. Os altos teores de ferro e alumínio reforçam a hipótese de que o alto teor de sílica possibilita identificar a origem predominantemente cerâmica do material RCD.

Tabela 3: Análises por FRX do Fíler RCD.

\begin{tabular}{ccccccccccc}
\hline \multirow{2}{*}{$\overbrace{}^{2}$} & \multicolumn{10}{c}{ \% DE ÓXIDOS (EM MASSA) } \\
\cline { 2 - 10 } & $\mathbf{S i O}_{\mathbf{2}}$ & $\mathbf{F e}_{2} \mathbf{O}_{\mathbf{3}}$ & $\mathbf{A l}_{\mathbf{2}} \mathbf{O}_{\mathbf{3}}$ & $\mathbf{K}_{\mathbf{2}} \mathbf{O}$ & $\mathbf{T i O}_{2}$ & $\mathbf{C a O}$ & $\mathbf{M g O}$ & $\mathbf{Z r O}_{2}$ & $\mathbf{S r O}$ & $\mathbf{M n O}$ \\
\cline { 2 - 11 } & $39,3 \%$ & $29,7 \%$ & $20 \%$ & $3,7 \%$ & $2,1 \%$ & $1,4 \%$ & $1,31 \%$ & $1,14 \%$ & $0,7 \%$ & $0,2 \%$ \\
\hline
\end{tabular}

\subsection{DOSAGEM MARSHALL}

\subsubsection{Análises das dosagens com parâmetros volumétricos da metodologia marshall}

Para os parâmetros estabelecidos de Estabilidade versus \% EAP (Figura 4), foi possível identificar maiores estabilidades dos corpos de prova (CP's) nas misturas D2 - BNAR, que representa a mistura de agregados entre brita natural e areia reciclada, utilizando a dosagem com teor de EAP em 6\% do total de material da mistura. Por meio da obtenção do gráfico Estabilidade versus \% EAP, constatou-se também que, na maioria das dosagens, quando é aumentado o teor de ligante na mistura percebe-se uma diminuição da estabilidade. Pode-se considerar a exceção da dosagem D1 - BNAN (agregados naturais) que em teores de $8 \%$ resultou em uma estabilidade alta quando comparada as demais dosagens.

Comparando a estabilidade do CP de concreto asfáltico utilizando o PMF com a do CP que faz uso de cimento asfáltico de petróleo (CAP) como ligante asfáltico e agregados integralmente naturais se identificam 
diferenças significativas nos resultados de estabilidade Marshall. De acordo com COSTA FILHO []ㅡ, em teores de CAP variando entre 5\% e 5,5\% a estabilidade do CP chega à faixa de 600 a $800 \mathrm{kgf}$, o que torna mais resistente o pavimento rodoviário quando sujeito a ações de agentes físicos externos (altas temperaturas, cargas elevadas, entre outros fatores).

Outro fator importante para a análise de resultados de misturas betuminosas é a identificação de volume de vazios ( $\mathrm{Vv}$ ) na mistura com maiores ou menores teores de ligante asfáltico (Gráfico Volume de vazios versus $\%$ EAP - Figura 4). Dessa forma, nas misturas betuminosas do tipo Pré-Misturado a Frio torna-se possível prever o quanto o teor de emulsão na mistura interfere na estabilidade e fluência mecânica da camada de revestimento do pavimento, observando, como consequência, o aumento ou diminuição do volume de vazios.

Em análise ao gráfico da relação de betume de vazios (RBV) versus \% de EAP - Figura 4, percebe-se também que os valores menores de RBV ocorreram em teores baixos de \% EAP, e os maiores valores de RBV se deram em teores mais elevados de \% EAP. Em posse dessas informações, e com base na análise dos resultados, pode-se inferir que nas condições de misturas investigadas com o PMF, o valor de RBV ótimo deve manter-se com percentuais de EAP nem excessivamente baixos, nem excessivamente altos. Essa conclusão pode ser relacionada com os ensaios de estabilidade realizados na pesquisa, pois com altos teores de EAP na dosagem o pavimento poderá sofrer deformações permanentes causando perda de estabilidade BERNUCI et al. [2].

De acordo com COSTA FILHO [3], outro fator impactante com a baixa relação betume vazios é a facilidade de infiltração de água na camada de revestimento do pavimento. Nesse caso, o concreto asfáltico fica susceptível a desfragmentação pela influência da presença de água prejudicando, dessa forma, a durabilidade do pavimento asfáltico.

Para a análise do gráfico da densidade aparente (Gmb) versus \% de EAP - Figura 4, tendo em vista os valores aproximados para a densidade aparente, foi possível constatar que com o aumento dos teores de \% EAP os valores da densidade se mantiveram constantes com uma tendência a leves diminuições. De acordo com COSTA FILHO [3], apesar dos valores serem aproximados, com o aumento de EAP na dosagem espera-se que a tendência das curvas do gráfico Gmb versus \% EAP tenha tendências ao aumento da densidade com a elevação do ligante, o que pode ser justificado pelo gráfico RBV versus \%EAP, pois nesse gráfico interpretou-se que o aumento de emulsão provoca um menor volume de vazios, o que consequentemente provocaria um concreto asfáltico com estrutura interna com menor índice de vazios.

Analisando os parâmetros de índices de vazios com a estabilidade medida, foi possível observar a diminuição da estabilidade (com semelhanças de comportamento tanto na concepção de misturas de agregados naturais tanto quanto nas misturas e RCD) quando tem-se a diminuição do volume de vazios. Essa análise justifica-se por meio da Figura 4 (Estabilidade versus \% de EAP) que com o aumento do teor de emulsão na mistura obtém-se a diminuição da estabilidade do CP. 
Estabilidade versus \% EAP

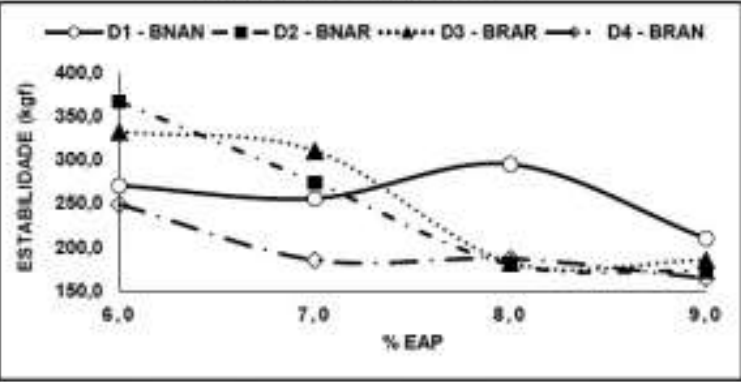

Volume de Vazios (Vv) versus \% EAP

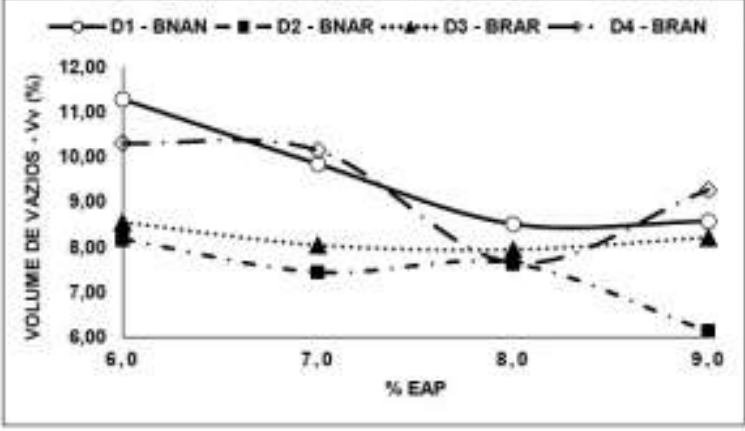

Relaçăo Betume-Vazios (RBV) versus \% EAP

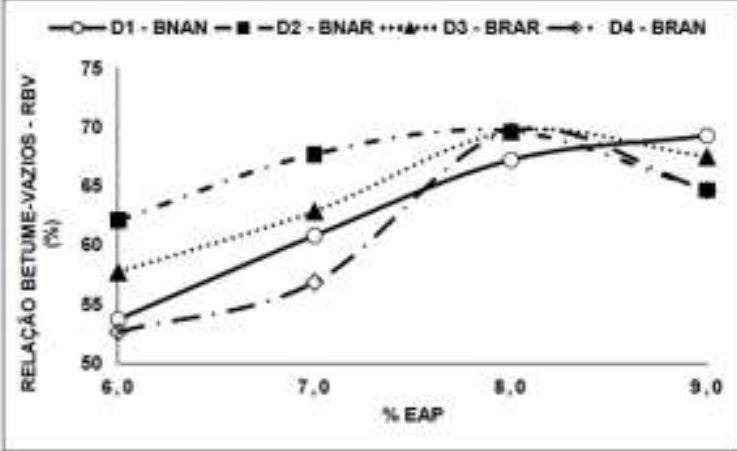

Densidade Aparente (Gmb) versus \% EAP

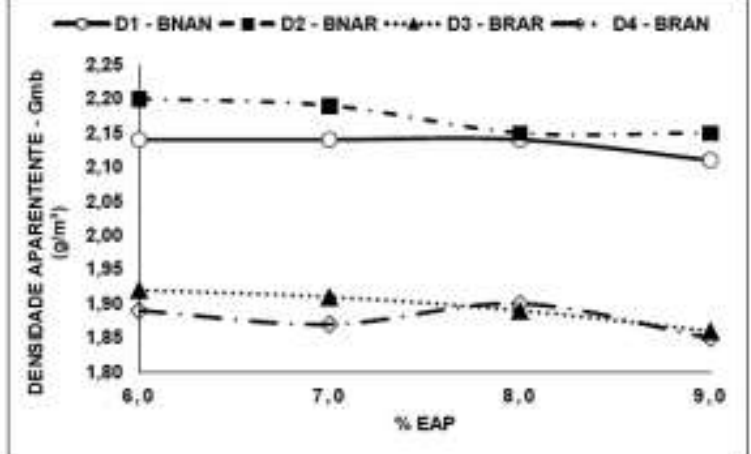

Figura 4: Gráficos referentes às análises de parâmetros de dosagens Marshall.

Analisando os parâmetros de índices de vazios com a estabilidade medida, foi possível observar a diminuição da estabilidade (com semelhanças de comportamento tanto na concepção de misturas de agregados naturais tanto quanto nas misturas e RCD) quando tem-se a diminuição do volume de vazios. Essa análise justifica-se por meio da Figura 5 (Estabilidade versus \% de EAP) que com o aumento do teor de emulsão na mistura obtém-se a diminuição da estabilidade do CP.

Avaliando os resultados que apontaram a diminuição do volume de vazios ( Vv) com a diminuição da estabilidade (Gráfico Estabilidade versus Volume de vazios Vv - Figura 5), esse fato pode está diretamente relacionado ao aumento do teor de emulsão na mistura, pois sendo esse um líquido fluído e viscoso, torna-se possível uma melhor compactação com um maior envolvimento dos agregados, diminuindo, consequentemente, o volume de vazios.

Diante de outra análise comparativa de parâmetros volumétricos, tornou-se importante analisar o grau de influência entre a estabilidade do concreto asfáltico e a relação betume-vazios (RBV). Conforme interpretação ilustrada por meio do gráfico Estabilidade versus RBV - Figura 5, nota-se que com aumento do percentual de RBV, ocorre a diminuição da estabilidade. Vale destacar ainda que entre as quatro concepções de mistura os comportamentos gráfico são semelhantes difenrindo-se, siginificatimente, na faixa de valores dos resultados de estabilidade, pois, conforme ensaios de caracterização física e mineralógica, os CPs que mantiveram presente os agregados graúdos naturais tiveram estabilidade superior quando comparado aos agregados graúdos com RCD.

Portanto, para os resultados da interação estabilidade versus RBV, conforme foi explicitado em análises do gráfico Estabilidade versus Vv, é importante estimar previamente um teor de ligante ótimo para compor a mistura entre os agregados. Dentro desse contexto, quantidades excessivas de emulsão na mistura, mesmo reduzindo o índice de vazios, pode ocasionar deformações permanentes no concreto com maiores facilidades; no entanto, baixos teores de emulsão aumentam o índice de vazios e podem diminuir a durabilidade do pavimento com o enfraquecimento da mistura e interferência direta nas propriedades de desempenho mecânico.

Com relação às análises de densidade aparente da mistura compactada com agregados naturais e RCD, observou-se variações próximas quando comparadas as quatro concepções de agregados e sem tedências a diminuição em dosangens altas de EAP (Gráfico Estabilidade versus Gmb - Figura 5). De forma tendenciosa, as curvas desse gráfico demonstram o aumento da estabilidade com o aumento do Gmb. Essa análise pode ser corroborada com o fato de que um concreto asfáltico de matriz densa, com baixo volume de vazios e agregados 
de perfeita formação estrutural influi positivamente na resistência mecânica, e consequentemente na sua durabilidade.

Na Figura 5 também tem-se a reprensentação da interação de resultados entre RBV com o Vv (Gráfico RBV versus Vv). Para tanto, a Figura demonstra um comportamento da seguinte forma: diminuindo-se a relação betume-vazios, aumenta-se o volume de vazios. Esse acontecimento é justifcado conforme os gráficos de $\mathrm{Vv}$ versus \% EAP (Figura 4). Com referência a esse gráfico, uma maior quantidade de ligante na mistura provoca um menor volume de vazios, o que nem sempre oportunizará uma boa estabilidade (Figura 5 - Estabilidade versus RBV). Para que se obtenha altas estabilidades do concreto asfáltico é necessário que seja feita uma dosagem de teor de emulsão ideal para não comprometer as propriedades físicas do pavimento.

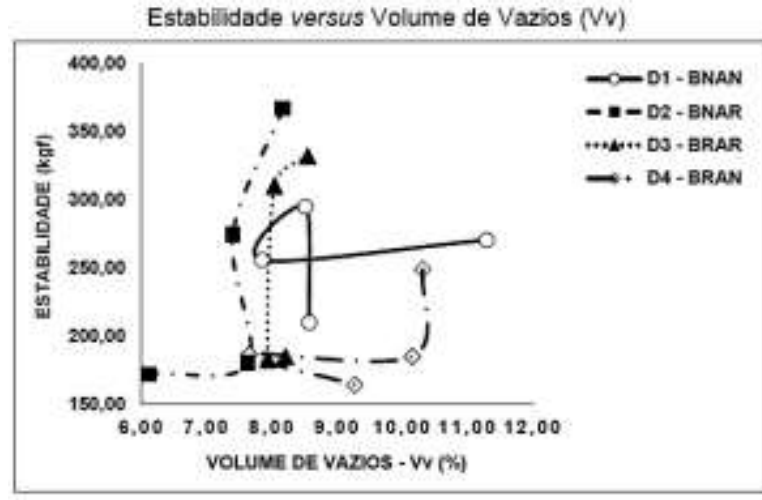

Estabilidade versus Relação Betume-Vazios (RBV)

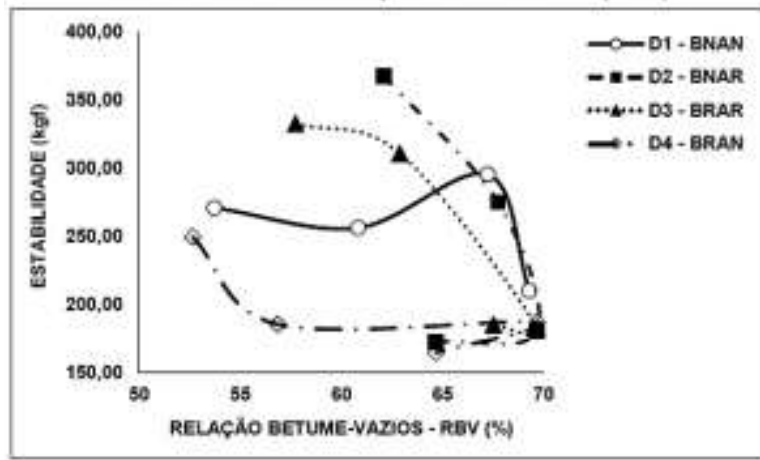

Estabilidade versus Densidade Aparente (Gmb)

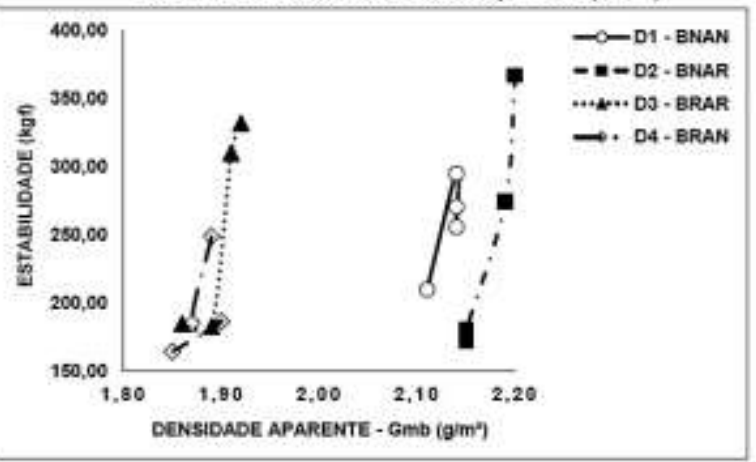

Relaçశ̊ Betume Vazios (RBV) versus Volume de vazios (VV)

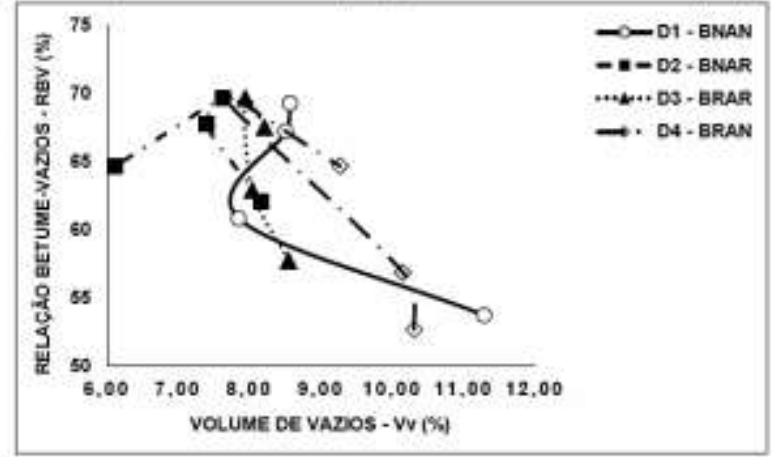

Figura 5: Gráficos referentes às análises de resultados das dosagens Marshall.

\subsubsection{Análises das dosagens com parâmetros da metodologia Marshall (Valores otimizados)}

A Figura 6 mostra os maiores valores de Estabilidade versus \% de EAP entre as quatro concepções de dosagens. Vale destacar que as dosagens que mantiveram os agregados integralmente naturais tiveram maiores valores de estabilidade com os percentuais de EAP entre $8 \%$ e $9 \%$. Entretanto, o maior valor de estabilidade foi com o teor de emulsão em 6\% e com a dosagem D2 - BNAR (Britra natural e areia reciclada). A dosagem que manteve na mistura os agregados integralmente de RCD obteve um valor máximo de estabilidade com teor de EAP em torno de 7\%. Diante desses resultados, pode-se inferir que a brita natural tem destaque no parâmetro estabilidade em maiores teores de ligante pelo motivo de ser um agregado de matriz granulométrica densa, com menor porosidade e maior resistência ao desgaste físico. Essas jutificativas são confirmadas por alguns ensaios de caracterização física e mineralógica.

No gráfico de pontos máximos da estabilidade versus RBV (Figura 6) foi possível constatar as seguintes análises: com o aumento da relação betume-vazios tem-se a diminuição da estabilidade e a diminuição do volume de vazios. Porém, dando ênfase as concepções de agregados das dosagens, as misturas que mantiveram presente a brita natural obteveram-se os maiores valores de estabilidade diferindo-se apenas a dosagem D3 BRAR. A dosagem que manteve agregados integralmentes reciclados, e em prováveis condições favoráveis de compactação, quando comparado com os demais CPs confeccionados a D3 - BRAR atingiu o maior valor de estabilidade.

Em pontos de valores máximos de Estabilidade versus Vv indicados nos gráficos da Figura 6, é possível constatar que com aumento do volume de vazios tem-se a diminuição da estabilidade. Logo, é possível afirmar 
que nos quatro diferentes teores de EAP analisados os agregados naturais predominantemente obtiveram os maiores valores de estabilidade, com excessão da dosagem D3 - BRAR que de forma excepcional atingiu o maior valor de estabilidade com o teor de 7\%. Tomando-se como referência as análises gráficas anteriores, esse acontecimento pode justificar-se pela possibilidade dos procedimentos de confecção de corpos de prova (em teor de $7 \%$ ) ocorrer de maneira mais favorável em relaçao aos demais.
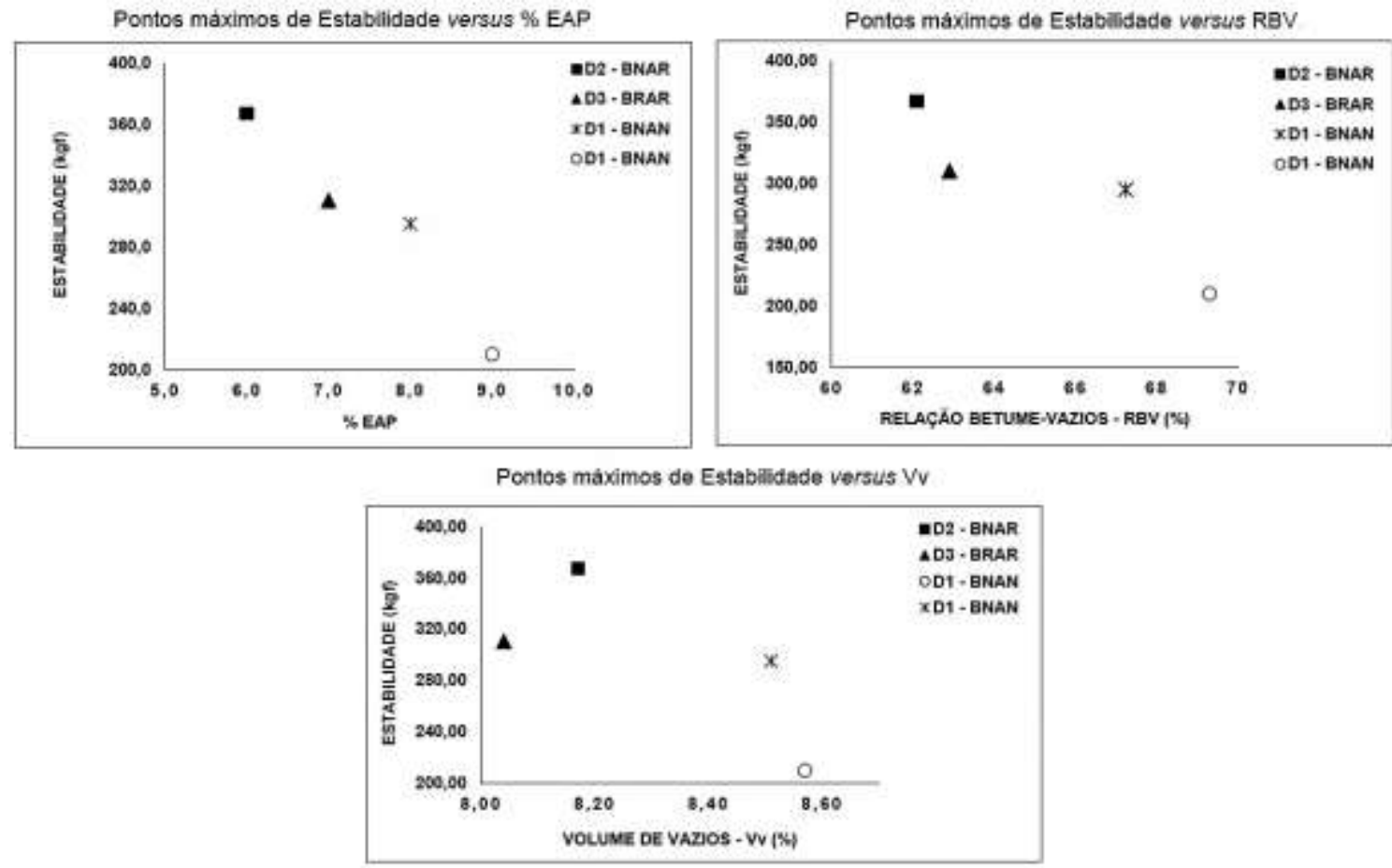

Figura 6: Gráficos referentes aos principais resultados de dosagens Marshall (otimizados).

\subsection{Traços otimizados para trecho experimental}

Viabilizando a possibilidade de uma futura análise em escala de campo e a execução de um trecho experimental para a continuidade desta pesquisa, os resultados dos gráficos que avaliaram a influência com parâmetros Marshall apontaram os melhores traços (otimizados) das dosagens, para a produção de concreto asfáltico com o PMF (Tabela 4).

Tabela 4: Dosagens otimizadas para trecho experimental após análises gráficas.

\begin{tabular}{ccccc}
\hline & $\begin{array}{c}\text { Dosagem 1 } \\
\text { Natural }\end{array}$ & $\begin{array}{c}\text { Dosagem 2 } \\
\text { Natural (Brita) } \\
\text { RCD (Areia) }\end{array}$ & $\begin{array}{c}\text { Dosagem 3 } \\
\text { RCD }\end{array}$ & $\begin{array}{c}\text { Dosagem 4 } \\
\text { RCD (Brita) + } \\
\text { Natural (Areia) }\end{array}$ \\
\hline \% Emulsão & $7,75 \%$ & $7,25 \%$ & $7,75 \%$ & $7,50 \%$ \\
\% B1 & $33,21 \%$ & $33,39 \%$ & $33,21 \%$ & $33,30 \%$ \\
\% B0 & $41,51 \%$ & $41,73 \%$ & $41,51 \%$ & $41,62 \%$ \\
\% Areia & $16,60 \%$ & $16,69 \%$ & $16,60 \%$ & $16,65 \%$ \\
\% Cimento & $0,92 \%$ & $0,92 \%$ & $0,92 \%$ & $0,92 \%$ \\
\hline Total & $\mathbf{1 0 0 \%}$ & $\mathbf{1 0 0 \%}$ & $\mathbf{1 0 0 \%}$ & $\mathbf{1 0 0 \%}$ \\
\hline
\end{tabular}




\section{CONCLUSÕES}

Diante da realização de todo o programa experimental desse trabalho, das análises dos resultados dos ensaios e com as respectivas discussões referentes aos estudos semelhantes já concretizados, foi possível obter as seguintes conclusões:

Verificou-se que grande parte das características físicas identificadas com o MEV tendem a favorecer numa melhor estabilidade e aderência superficial no concreto asfáltico avaliado, o que culmina num produto de potencial elevado para uso em camadas de revestimentos de pavimentos viários.

Com o ensaio de EDS e FRX foi possível identificar grandes percentuais de ferro e alumínio, que caracterizam os agregados oriundos do RCD como um material que contém grandes percentuais de frações cerâmicas presente no lote. Por conseguinte, a utilização dos agregados contendo o RCD em análise se tornaria viável em camadas de revestimentos em rodovias de baixo tráfego e de veículos leves, de preferência.

No ensaio de DRX, com a identificação de calcita, quartzo e caulinita, confirmam-se os resultados obtidos nos demais ensaios, destacando a presença predominante de sílica, alumínio, ferro e cálcio, os quais caracterizam o material de origem cerâmica.

Nas dosagens Marshall, a título de comparação gráfica dos resultados, os gráficos que mediram as interações dos resultados apontaram maior estabilidade com um menor Volume de Vazios (Vv) e maior densidade aparente $(\mathrm{Gmb})$ com menores percentual de EAP, pois o percentual ótimo de EAP na mistura favorece para um eficiente empacotamento dos grãos e, consequentemente, uma matriz densa.

Analisando os valores gráficos dos resultados otimizados das dosagens Marshall, constatou-se que os corpos de prova que contiveram agregados graúdos de origem natural, na sua composição, obtiveram resultados mais eficientes quando se verificou a perda de estabilidade. Entretanto, analisando o percentual de EAP e a Relação Betume-Vazios, percebeu-se que com o teor ótimo de EAP na mistura obteve-se resultados de estabilidade mais eficientes, como também, com uma menor Relação Betume-Vazios, obteve-se maiores valores de estabilidade.

Em relação aos resultados das dosagens Marshall, conforme a obtenção dos gráficos e tomando como base as comparações realizadas entre duas ou mais variáveis, pode-se inferir que os teores de ligante ótimos encontrados para as dosagens D1 - BNAN, D2 - BNAR, D3 - BRAR e D4 - BRAR foram, respectivamente, $7,75 \%, 7,25 \%, 7,75$ e $7,5 \%$.

Portanto, em posse de todos os resultados da pesquisa, espera-se que com uma destinação ambientalmente correta para os resíduos da construção e demolição, se tenha a proposta de aplicações dos agregados reciclados em pavimentos de concreto asfáltico, de maneira que se enquadre os resultados de ensaios nos limites estabelecidos em normas. Dessa forma, pode-se concluir que a aplicação dos resíduos de construção e demolição de obras como agregados reciclados em pavimentos de concreto asfáltico, de maneira que se enquadre nos limites estabelecidos nas normas vigentes, constitui uma destinação ambientalmente correta e viável para pavimentos de baixo volume de tráfego.

\section{AGRADECIMENTOS}

Os autores agradecem a Usina de reciclagem Duarte Ltda e a TcPAV Ltda pela doação dos materiais da pesquisa e ao Programa de Pós-Graduação em Engenharia Civil da Universidade Federal do Rio Grande do Norte e ao Instituto Federal de Educação, Ciência e Tecnologia do Rio Grande do Norte por todo o suporte da pesquisa.

\section{BIBLIOGRAFIA}

[1] ANTHONISSEN, J., BRAET, J., VAN, W. (2015) "Life cycle assessment of bituminous pavements produced at various temperatures in the Belgium context", Transportation Research Part D: Transport and Environment. Elsevier BV., pp. 306-317.

[2] BERNUCCI, L. B., MOTTA, L. M. G., CERATTI, J. A. P., et al., Pavimentação asfáltica: formação básica para engenheiros, Petrobras ABEDA, Rio de Janeiro, 2006.

[3] COSTA FILHO, F. C. "Estudo de viabilidade técnica do uso de resíduos oriundos do beneficiamento de Scheelita na composição de concretos asfálticos”, Dissertação de Mestrado, Universidade Federal do Rio Grande do Norte, Natal, 173 p, 2017.

[4] FATEMI, S., IMANINASAB, R. "Performance evaluation of recycled asphalt mixtures by construction and demolition waste materials", Construction and Building Material Elsevier BV, pp. 450-456, 2016. 
[5] GIMÉNEZ, A. M. G. "Estudo experimental de um resíduo de construção e demolição (RCD) para utilização em pavimentação", Dissertação de Mestrado, Universidade de Brasília, Brasília, 148 p, 2011.

[6] GÓMEZ-MEIJIDE, B., PEREZ, I., PASANDÍN, A, R. "Recycled construction and demolition waste in Cold Asphalt Mixtures: evolutionary properties”, Journal of Cleaner Production., pp. 588-598, 2016.

[7] JAMSHIDI, A., KURUMISAWA, K., NAWA, T., et al., "Characterization of effects of thermal property of aggregate on the carbon footprint of asphalt industries in China", Journal of Traffic and Transportation Engineering (english Edition), pp.118-130, 2017.

[8] LEITE, F. C., MOTTA, R. S., VASCONCELOS, K. L., et al., "Laboratory evaluation of recycled construction and demolition waste for pavements", Construction and Building Materials, pp.2972-2979, 2011.

\section{ORCID}

Plácido Gondim de Sena Neto

https://orcid.org/0000-0001-7997-506X

Enio Fernandes Amorim

https://orcid.org/0000-0001-7386-3956

Maria Del Pilar Durante Ingunza

https://orcid.org/0000-0001-6994-7559 